\title{
Raman Spectroscopy: Stimulated Raman Scattering Microscopy for Label-Free Chemical Imaging
}

\section{Citation}

Freudiger, Christian W., Wei Min, Brian G. Saar, and X. Sunney Xie. 2009. Raman spectroscopy: stimulated Raman scattering microscopy for label-free chemical imaging. Optics and Photonics News 20(12): 30.

\section{Published Version}

doi:10.1364/OPN.20.12.000030

\section{Permanent link}

http://nrs.harvard.edu/urn-3:HUL.InstRepos:11315422

\section{Terms of Use}

This article was downloaded from Harvard University's DASH repository, and is made available under the terms and conditions applicable to Open Access Policy Articles, as set forth at http:// nrs.harvard.edu/urn-3:HUL.InstRepos:dash.current.terms-of-use\#OAP

\section{Share Your Story}

The Harvard community has made this article openly available.

Please share how this access benefits you. Submit a story.

\section{Accessibility}




\title{
Stimulated Raman scattering (SRS) microscopy for label-free chemical imaging with high sensitivity
}

\author{
Christian W. Freudiger ${ }^{1,2, \star}$, Wei $\mathrm{Min}^{2, *}$, Brian G. Saar ${ }^{2}$ and X. Sunney $\mathrm{Xie}^{2}$ \\ ${ }^{1}$ Department of Physics, Harvard University, Cambridge (USA) \\ ${ }^{2}$ Department of Chemistry and Chemical Biology, Harvard University, Cambridge (USA) \\ * authors contributed equally
}

We recently developed a new multiphoton imaging technique, stimulated Raman scattering (SRS) microscopy, which allows chemical imaging of biological samples without the need for staining or fluorescent labeling. ${ }^{1}$ Such label-free imaging is desirable in biomedical research, because labeling often perturbs the function of small metabolite and drug molecules and may be too toxic to use in vivo in medical diagnostics.

The contrast of the technique originates from molecular vibrational frequencies that are intrinsic to specific chemical compounds. Imaging based on vibrational spectroscopy has traditionally relied on infrared absorption or spontaneous Raman scattering, which have limited utility for biomedical imaging because of low spatial resolution and slow imaging speed, respectively. In stimulated Raman scattering, however, the Raman signal is enhanced by orders of magnitude by virtue of stimulated excitation of molecular vibrational transitions, allowing for fast image acquisition. As in multiphoton-excited fluorescence, the nonlinear intensity-dependence of SRS also allows intrinsic three-dimensional sectioning capability.

Although stimulated Raman scattering microscopy had been reported by Ploetz et al., ${ }^{2}$ the high peak power lasers and low sensitivity were incompatible with biological applications. We developed a different approach: instead of boosting the nonlinear signal by using very high peak powers, we implemented a high-frequency phase-sensitive detection scheme to extract the week SRS signal that would otherwise be buried in laser noise of orders of magnitude higher amplitude. ${ }^{3}$ This approach allows for superb sensitivity at biocompatible excitation power levels.

Compared to the previous coherent Raman imaging technique, coherent anti-Stokes Raman scattering (CARS), ${ }^{4}$ SRS microscopy has the advantage that it does not suffer from the unwanted "nonresonant background" signal that limits sensitivity, distorts vibrational spectra and introduces imaging artifacts in CARS. As such, SRS is no longer limited to imaging of only the strongest Raman bands, and allows access to vibrations in the crowded fingerprint region of Raman spectra. The SRS signal is also linear in analyte concentration and free from complications related to phase-matching conditions. This allows for a more easilyinterpreted image contrast and makes coherent Raman imaging more accessible to the broader biomedical community.

We have demonstrated a variety of biomedical applications, including following the uptake of omega-3 fatty acids by living cancer cells and monitoring the transdermal delivery of topically applied drugs into mouse skin $^{1}$. The figure shows SRS images of the hydrophobic drug retinoic acid and the hydrophilic penetration enhancer dimethyl sulfoxide. We are able to visualize that the different compounds follow distinct penetration pathways into the skin, highlighting the potential of SRS microscopy to study pharamacokinetics.

The strongest SRS signals in biological samples originate from $\mathrm{CH}_{2}$ stretching vibration from lipids. They can be utilized for rapid imaging of tissue morphology without staining. ${ }^{5}$ As such, coherent Raman imaging can be used for in vivo virtual histology of fresh and unfixed tissue and for distinguishing healthy tissue from diseased or tumorous tissue. This will allow for minimally invasive optical biopsies based on coherent Raman imaging. 


\section{Figure}
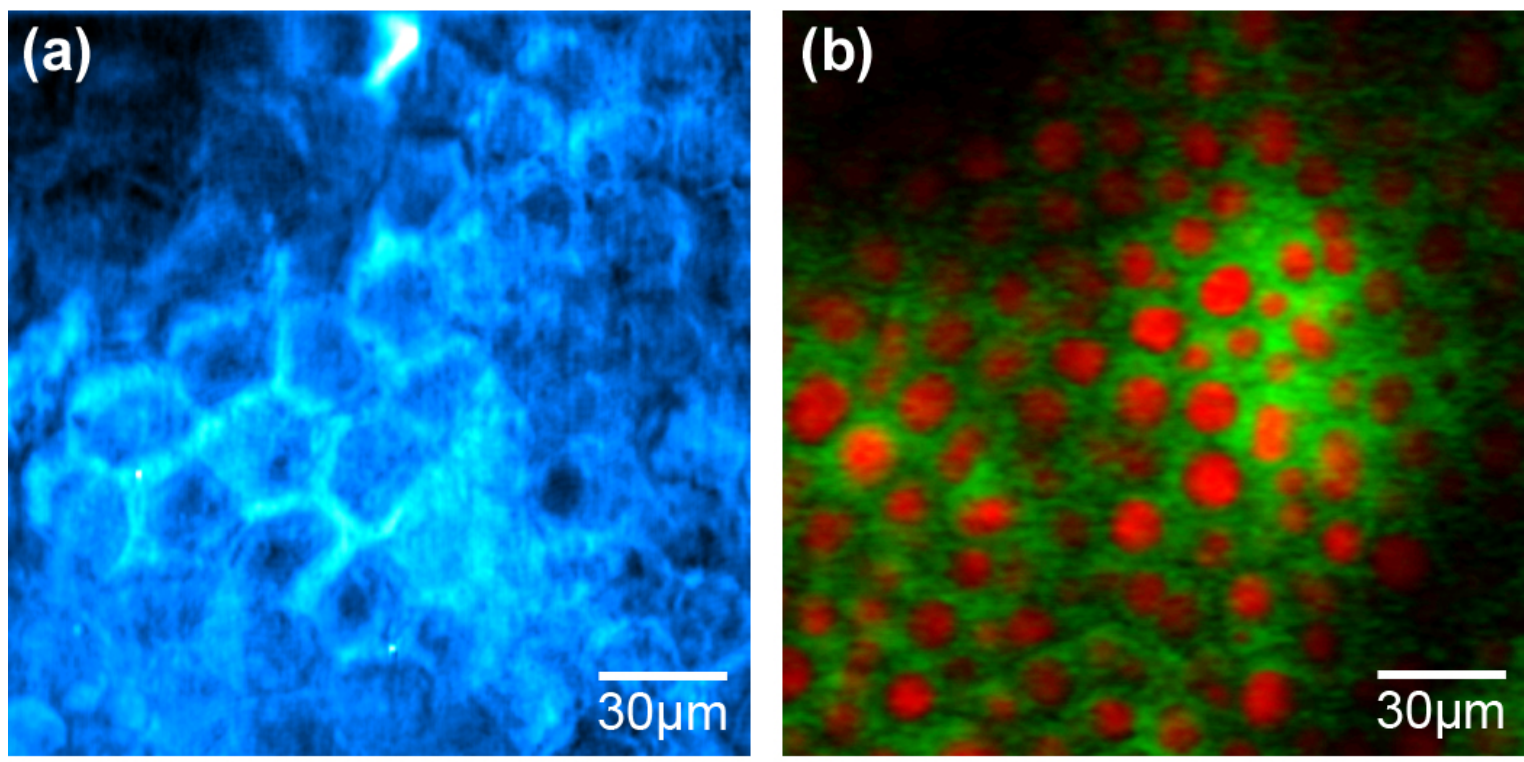

(c)
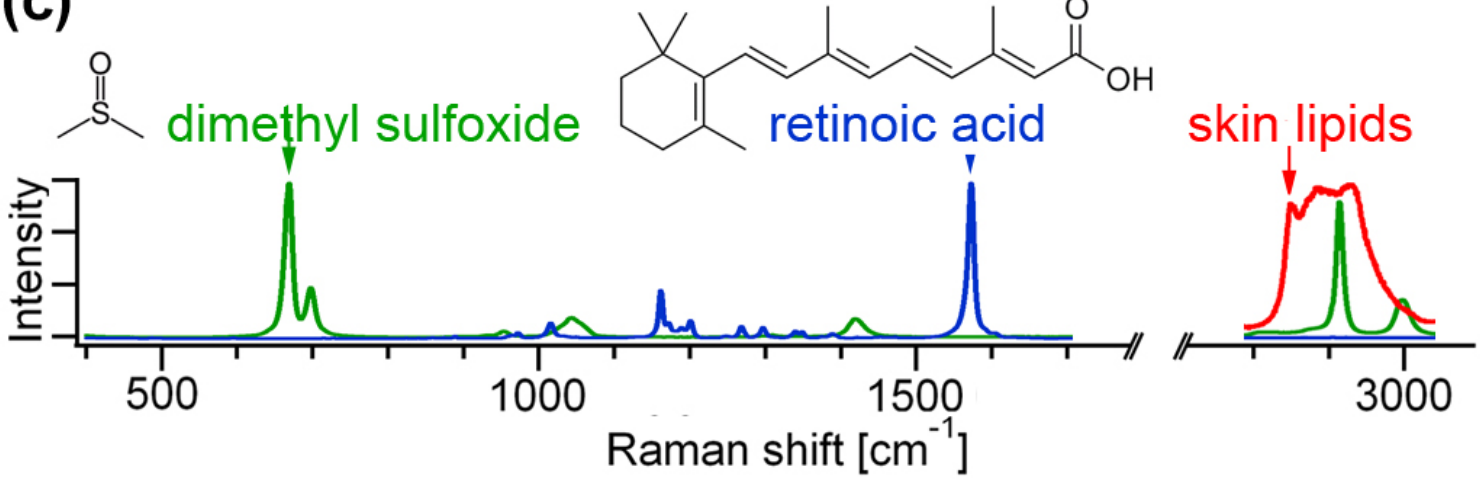

Label-free imaging of drug delivery. Lateral profile in mouse skin of (a) the topically applied compound retinoic acid (RA) and (b) the penetration enhancer dimethyl sulfoxide (DMSO). These images were acquired with SRS microscopy tuned into the Raman shifts (c) of RA at $1570 \mathrm{~cm}^{-1}$ (blue) and DMSO at $670 \mathrm{~cm}^{-1}$ (green). Skin structures are highlighted by tuning into the $\mathrm{CH}_{2}$ stretching vibration of typical skin lipids at $2845 \mathrm{~cm}^{-1}$ (red). The hydrophobic RA penetrates through the lipid-rich intercellular space of the stratum corneum in (a), while the hydrophilic DMSO avoids lipid-rich skin structures such as the adipocytes in the subcutaneous fat layer, as shown in (b).

\section{References:}

[1] C.W. Freudiger, W. Min et al. "Label-Free Biomedical Imaging with High Sensitivity by Stimulated Raman Scattering Microscopy," Science, 322, 1857-1861 (2008)

[2] E. Ploetz et al. "Femtosecond stimulated Raman microscopy," App. Phys. B 87, 389 (2007)

[3] B.F. Levine "Surface vibrational spectroscopy using stimulated Raman-scattering," IEEE J Quan. El. 15, 1418 (1979)

[4] A. Zumbusch et al. "Vibrational Microscopy Using Coherent Anti-Stokes Raman Scattering," Phys. Rev. Lett. 82, 4142 (1999)

[5] C.L. Evans et al. "Chemically-selective Imaging of Brain Structures with CARS Microscopy," Optics Express, 15, 12076-12087 (2007). 